\section{Characteristics of fine vascular network pattern associated with recurrence of polypoidal choroidal vasculopathy}

A Oishi' ${ }^{1}$ M Mandai ${ }^{1,2}$, M Kimakura ${ }^{1}$, A Nishida ${ }^{1}$ and $Y$ Kurimoto ${ }^{1}$
${ }^{1}$ Department of

Ophthalmology, Kobe City Medical Center General

Hospital, Kobe, Japan

${ }^{2}$ Laboratory for Retinal Regeneration, Center for Developmental Biology, RIKEN, Kobe, Japan

Correspondence: M Mandai, Laboratory for Retinal Regeneration, Center for Developmental Biology, RIKEN, 2-2-3 Minatojima-minamimachi, Chuo-ku, Kobe 650-0047, Japan

Tel: + 81783060111 ; Fax: + 81783060101

E-mail: mmandai@ cdb.riken.jp

Received: 10 February 2011 Accepted in revised form: 18 March 2011 Published online: 6 May 2011

\begin{abstract}
Objective To characterize an irregular capillary-like structure in the vascular network of eyes with polypoidal choroidal vasculopathy (PCV), and to determine whether its presence after photodynamic therapy (PDT) can be used to predict the clinical course of PCV.

Methods We reviewed the clinical records of 29 eyes of 29 patients with PCV, who underwent PDT and confocal retinal angiographic examinations every 3 months. The images obtained before the PDT were compared with those after the PDT. The correlations between angiography findings and recurrences were evaluated.

Results An area of fine, densely packed capillary-like vessels, named the fine vascular network, was identified within the polypoidal vascular network in 25 of 29 cases at the initial examination. The fine vascular network regressed in 23 cases $\mathbf{( 9 2 \% )}$ ) after the first PDT. Thereafter, the fine vascular network remained or enlarged in 19 eyes, and $17(84.5 \%)$ of these eyes had a recurrence of the polypoidal lesions or had exudative changes. In contrast, recurrences were found in only 2 of $10(20 \%)$ eyes, whose fine network had regressed without a subsequent enlargement $(P<0.001$ compared with the former group).

Conclusions A fine irregular vascular network is present in the majority of eyes with PCV before PDT. Its presence or expansion after PDT was significantly associated with a recurrence of PCV. Thus, we recommend that this network be monitored after treatment to determine whether a polypoidal vascular network will recur.
\end{abstract}

Eye (2011) 25, 1020-1026; doi:10.1038/eye.2011.110; published online 6 May 2011

Keywords: age-related macular degeneration; occult choroidal neovascularization; photodynamic therapy; polypoidal choroidal vasculopathy; vascular network

\section{Introduction}

Polypoidal choroidal vasculopathy $(\mathrm{PCV}){ }_{1}^{1}$ is either a subtype or a distinct type of age-related macular degeneration. PCV is characterized by a branching vascular network (BVN) with polypoidal lesions. Several therapeutic procedures are used to treat $\mathrm{PCV}$, including photodynamic therapy (PDT) and intravitreal injections of anti-vascular endothelial growth factor (VEGF). Patients with PCV generally respond well to $\mathrm{PDT} ;{ }^{2-11}$ however, the vascular network often persists and causes recurrences ${ }^{10,12}$ which prevent good visual recovery. Therefore, reducing the number of recurrences is an important aspect of the treatment of PCV. ${ }^{13,14}$

Although there is no established way to predict recurrences, the indocyanine green angiographic (IA) findings seemed to offer some clues as was suggested by Kang et al..$^{15}$ They investigated the staining pattern in late phase IA images and found a late geographic hyperfluorescent (LGH) pattern, which matched the total area of the branching network and polyps. They found that a persistence of the LGH pattern was associated with the recurrence of the polypoidal lesions. ${ }^{15}$

To further explore the characteristic IA findings associated with the vascular pattern in 

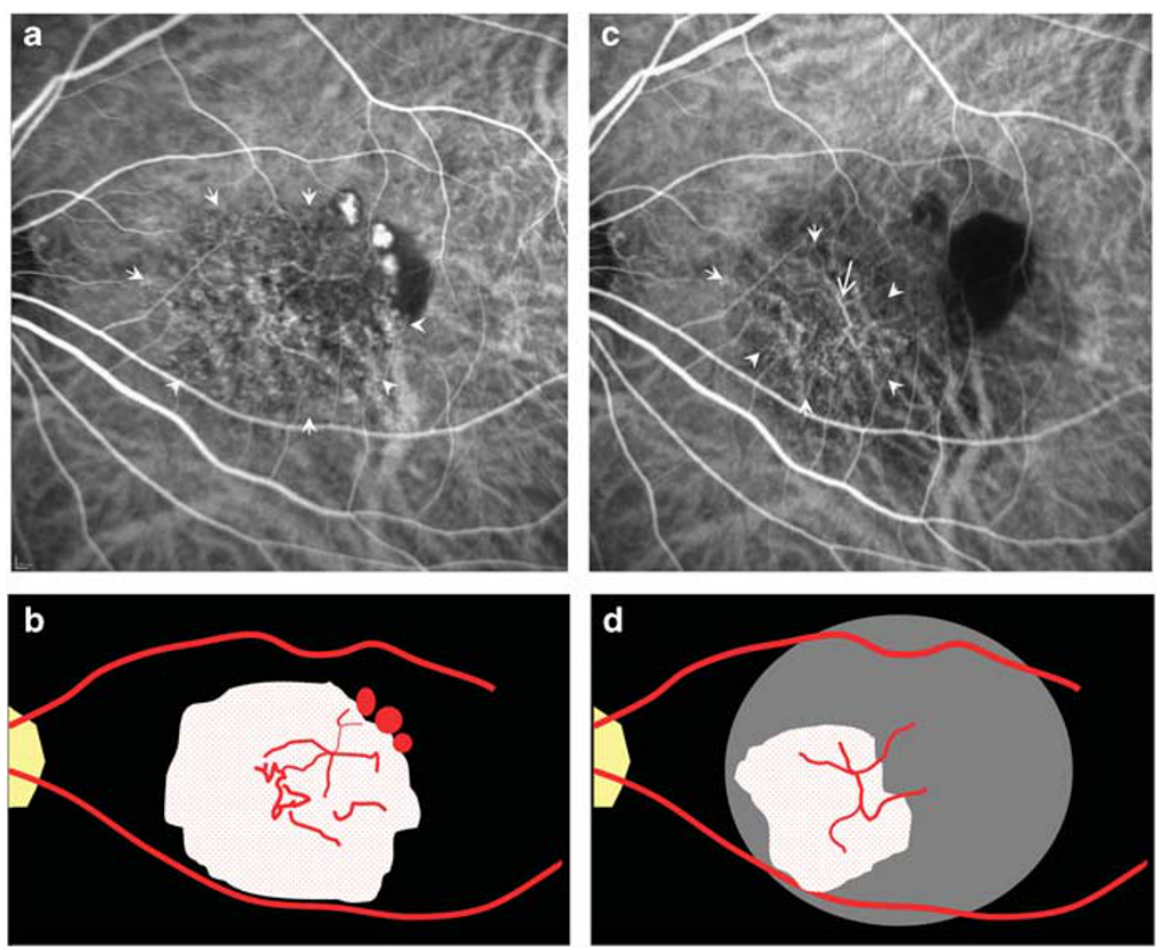

Figure 1 IA images at 80-90s after the injection of indocyanine green in patients with PCV. (a) Image of a pre-treatment eye. Arrowheads indicate the area of the fine vascular capillary-like network. The fine vascular network consists of the fine crowded vessels sometimes surrounding a relatively large trunk. Polypoidal lesions are confirmed at the terminals of the fine vascular network. (b) Schematic image showing the fine vascular network vessels. The dotted area represents what we consider the fine vascular network. Relatively large trunks are seen in the area. (c) IA image of the same eye after PDT. Trunks, which are often referred to as a branching vascular network, are prominent in this image (arrow). PDT irradiated area frequently shows a hypofluorescent area as in this case. The persistent fine vascular network can be seen in the hypofluorescent area. (arrowhead). (d) Schematic image of the area of the fine vascular network (dotted area), large vessels, and hypofluorescent area (gray area).

eyes with PCV, and to determine whether the pattern had a predictive value on recurrences, we reviewed the early to middle phase IA images of eyes with PCV that had undergone PDT. In the middle phase IA images (1-1.5 min) before treatment, the PCV vascular network contained an area that consisted mainly of fine and densely packed vessels, and individual vessels were difficult to detect. After treatment or stabilization, the fine vascular network often regressed, and larger welldemarcated vessels with diameters larger than $30 \mu \mathrm{m}$ became visible (Figure 1). We considered that the former vascular structures corresponded to the irregular choroidal capillary network or to neovascular structures representing newly developed or an active component, and the latter choroidal arteriole/venule-like structures represented a more differentiated stabilized structure.

The purpose of this study was to determine whether the fine vascular structure represented some pathological state and would therefore be found more frequently in newly developed or active BVN. We named this capillary-like component within the BVN the 'fine vascular network,' and investigated whether its presence can predict a recurrence of the PCV.

\section{Subjects and methods}

We reviewed the clinical records of 29 eyes of 29 patients who had undergone PDT between November 2005 and August 2008, and were followed with angiographic examinations every 3 months for at least 1 year.

We certify that all applicable institutional and governmental regulations concerning the ethical use of human volunteers were followed during the treatment of the patients and in this research.

Fluorescein angiography and IA were performed by confocal scanning retinal angiography with the HRA2 (Heidelberg Engineering, Dossenheim, Germany), before and every 3 months after the treatment. The presence and change in the size of the fine vascular network present within the polypoidal vaculature was determined in the IA images obtained 60-90 s after the injection of indocyanine green. Fluorescein angiography and optical 



Figure 2 IA images of a 61-year-old man with PCV. (a) Pretreatment IA image at an early phase showing a distinct vascular network and polypoidal lesions at their terminals. The entire branching vascular network appears as a fine vascular network (arrowheads). (b) IA in late phase showing LGH. The area corresponds approximately to that of the fine vascular network (arrowheads). (c) After 3 months of the first PDT. Some polypoidal lesions have regressed and the fine vascular network is less distinct (arrowheads). In contrast, the arteriole-/venule-like vessels appear more prominent (arrow). (d) After 6 months of the PDT. The fine vascular network is still present and has enlarged downward (arrowheads). (e, f) After 9 months of the PDT. New polypoidal lesions appear along the edge of the enlarged fine vascular network (arrows). The entire area of the fine vascular network is indicated by the arrowheads. Fine vascular network has enlarged, and the LGH has also enlarged (arrowheads). The staining is more uniform in the newly developed fine vascular network.

coherence tomography (OCT) were used to detect a recurrence of the polypoidal lesions. The OCT images were obtained by either the OCT1000 (Topcon, Tokyo, Japan), or the Spectralis OCT (Heidelberg).

PDT with verteporfin (Visudyne; Novartis AG, Basel, Switzerland) was carried out with a 689-nm laser system (Carl Zeiss, Dublin, CA, USA) using the standard protocol, and the area irradiated was determined from the IA images. ${ }^{9,16}$ When exudative changes, for example, retinal edema and subretinal fluid, were observed in the FA or IA images, or in the OCT images, the eyes were retreated.

The SPSS (ver 11.0.1; SPSS Japan, Tokyo, Japan) statistical program was used to analyze the data. $\chi^{2}$ tests were used to determine whether the differences were significant. Descriptive analyses are reported as the means \pm SDs, unless otherwise specified.

\section{Results}

A fine vascular network was observed within the polypoidal vascular network in 25 of 29 eyes in the pre-PDT images. In the other four patients, a prominent pigment epithelial detachment (PED), or a subretinal hemorrhage prevented a detailed examination of the IA images. The fine network decreased in size in 23 of the 25 cases within 6 months after the initial PDT. The fine vascular network was detected in two of the previously undetermined four cases after the resolution of the PED, or subretinal hemorrhage. Thus, 27 of the 29 cases had a fine vascular network within the polypoidal lesion before the PDT.

During the follow-up, 19 of the 27 patients had a recurrence or enlargement of the fine vascular network. Among these 19 patients, a recurrence of exudative changes and polypoidal lesions were observed in 17 and 15 patients, respectively (Figure 2). The enlargement of the fine vascular network preceded the appearance of the polypoidal lesions in four of the 15 patients. When the fine vascular network enlarged, the corresponding LGH was also enlarged, and the hyperfluorescence was generally uniform over the fine vascular network.

In the other 10 patients, the fine vascular network continued to decrease. A hypofluorescent area surrounding the more prominent arteriole-/venules-like vessels was often observed in these patients. None of these patients had a recurrence of the polyps, and only two developed exudative changes without polyps. When the fine network regressed, the LGH also became indistinct. (Figure 3) There was a significant difference in 

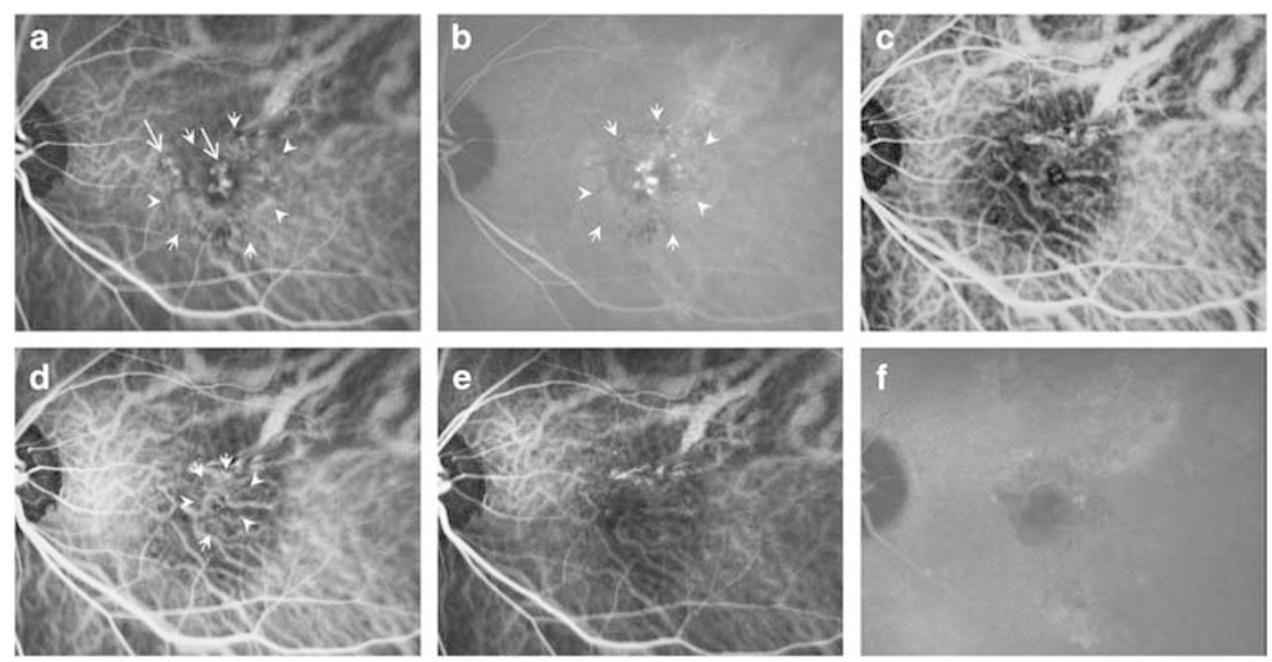

Figure 3 IA images of a 65-year-old man with PCV. (a) Pre-treatment IA showing fine vascular networks (arrowheads), and polypoidal lesions (arrows). (b) Late phase IA shows LGH corresponding to the fine vascular network (arrowheads). (c) IA image after 3 months of the PDT. The vessel structure has become evident with regression of the fine vascular network in response to the PDT (arrowheads). The polypoidal lesion is also reduced, but is not accompanied by exudative changes. (d, e) After 6 (d) and 12 months (e) of PDT. The fine vascular networks have decreased and become indistinct. This case did not have a recurrence. (f) IA image showing that LGH also becomes indistinct as the fine vascular network regressed.

the recurrence rates of those with and without an enlargement of the fine vascular network $\left(P<0.01, \chi^{2}\right.$-tests).

We also used OCT to monitor the eyes, although quantitative measurements were not performed, because of the difficulty of making point-to-point comparisons. In the OCT images, the fine vascular network usually appeared as a flat to moderate high PED, which may correspond to the reported double layer sign. ${ }^{17}$ In some cases, the double layer sign became flat as the fine network regressed, or became thicker when the fine network re-appeared (Figure 4). A complete resolution of the fine vascular network was rarely observed despite a decrease of its thickness.

\section{Discussion}

The network vessels in eyes with PCV appear heterogeneous in the IA images. The pre-PDT lesion often contained a fine, mesh-like irregular hyperfluorescent pattern in the images obtained in the middle phase of ICG angiography. Following PDT, the fine vascular network often became less evident and larger vessels became more prominent. These larger vessels resembled choroidal arterioles and venules; whether these vessels are a part of the choroidal vessels or differentiated neovascular vessels was not determined. The term BVN is commonly used in describing eyes with PCV, and refers to the whole network area, or these relatively large vessels. Several studies have reported that the BVN did not disappear after treatment. ${ }^{10,12,18}$ However, if we closely investigated the detailed characteristics of BVN, the post-treatment changes seemed to occur mainly in the fine vascular network. The presence of the fine vascular network within the BVN seemed to behave as an indicator of the disease activity. Thus, the aim of this study was to determine the significance of the fine vascular network.

We studied the recurrence or regrowth of the fine vascular network after PDT, and found that eyes with an enlargement of the fine vascular network were associated with recurrences of the exudative changes or polypoidal lesions. The frequency of fine vascular networks was especially high in untreated eyes. In fact, 25 of 29 untreated eyes had a distinct fine vascular network, that was virtually all the eyes without blocked fluorescence by PED or dense hemorrhage.

Arteriole-/venule-like vessels were also observed in untreated eyes, but they were more evident in the post-PDT stabilized eyes. Also, the fine vascular network frequently reappeared, accompanied by a recurrence of the polypoidal lesion. In fact, the presence of fine vascular network often preceded a clinically significant recurrence with accumulation of sub-epithelial or sub-retinal fluid and occasionally polyp formation.

These findings suggest that the fine vascular network may represent the active component of the PCV lesion. Observations of the corresponding uniform hyperfluorescent area within the late staining pattern $(\mathrm{LGH})^{15}$ in the area of the fine vascular network may indicate a relative leakiness of these crowded vessels, 

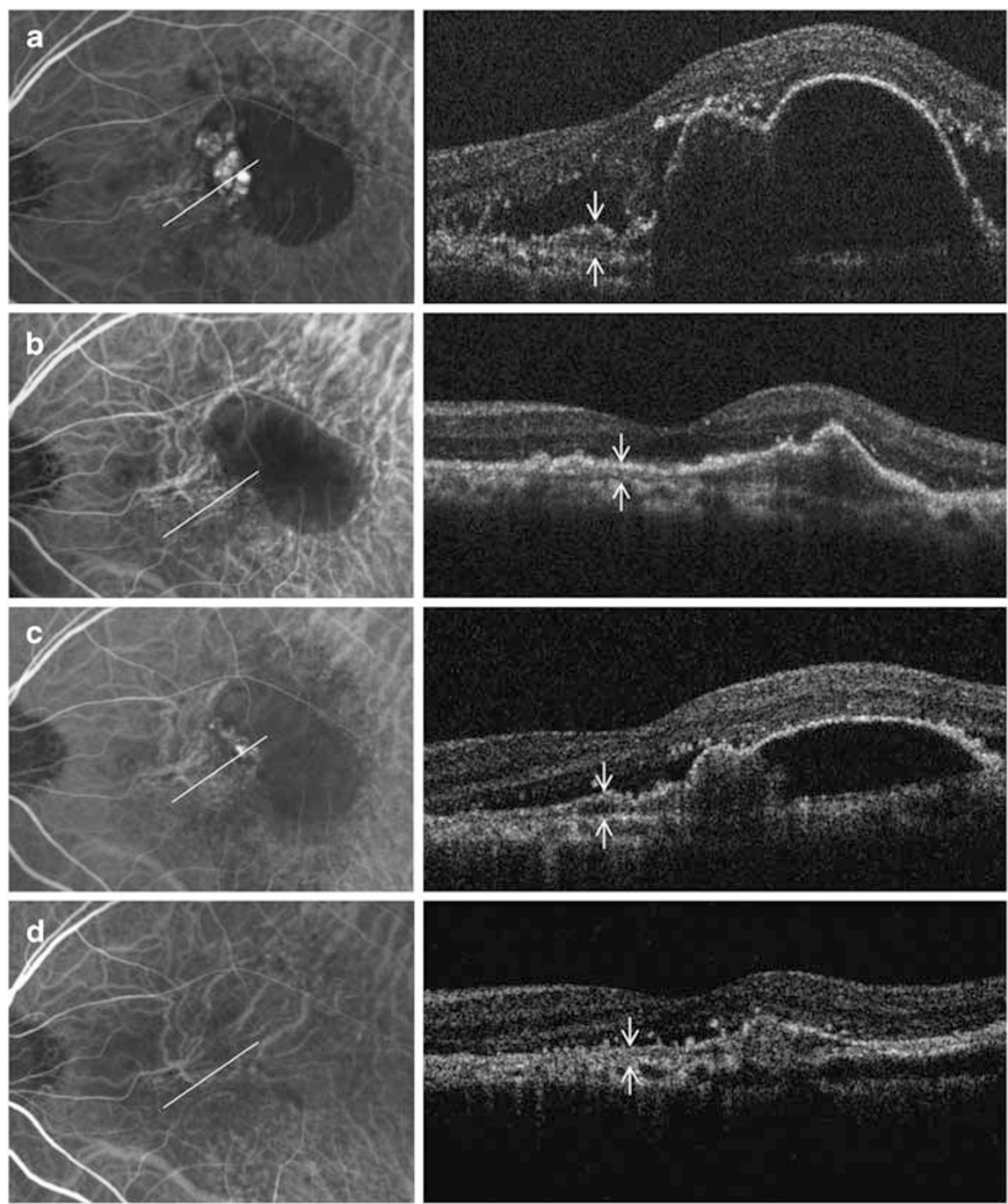

Figure 4 IA and corresponding OCT images of a 65-year-old patient with PCV, treated with PDT. (a) Pre-treatment OCT of polypoidal lesion showing prominent PED. Fine vascular network with flat PED (arrows) can be seen adjacent to the polypodidal lesion. (b) After PDT and regression of the fine vascular network, the PED becomes flat (arrows). (c) IA image showing a recurrence of the fine vascular network and polypoidal lesion. The height of the PED is increased (arrows). (d) After the retreatment, the PED is flattened again.

compared with the background choroidal capillary beds. This suggests the presence of an ectopic fine vascular network, that is, choroidal neovascularization.

On the other hand, the LGH tended to become more obscure after the regression of the fine vascular network. However, the LGH was not detected as frequently in our eyes as Kang et al reported. Thus, the relationship between LGH and fine vascular network is yet to be determined, but we assume that the findings would be complementary to our observations of the fine vascular network in evaluating the activity of the polypoidal lesion.

The appearance of the fine vascular network in the OCT images has some important implications. The OCT images usually showed flat to moderate PED (double layer sign $)^{17}$ in the area of the fine vascular network. In typical fresh cases, the double layer sign is lost when the fine vascular network regresses or increases when the network enlarges. However, the double layer sign rarely disappears even after the regression, especially in longstanding cases associated with fibrovascular PED. These findings suggest that the abnormal choroidal vessels under Bruch's membrane may permit fluid to leak into the sub-pigment epithelial space with the impairment of the membrane in the early phase. ${ }^{19,22}$ However, in majority of the cases, the fine vascular network seemed to be present within or above Bruch's membrane, and may permit fluid leakage into the sub-pigment epithelial space. This would then be compatible with the reported location of the PCV network and polyps. ${ }^{20-24}$ 
Because we were interested in the differences in the components of the vascular structures after different types of treatments, we also reviewed the images of eyes that had been treated with anti-VEGF therapy. In a pilot case review, we found that the fine vascular network usually persisted without any evidence of regression in patients treated by anti-VEGF therapy, which is consistent with a recent report. ${ }^{18}$ This difference in the response to the anti-VEGF therapy may be one reason for the low rate of polyp regression after anti-VEGF therapy for $\mathrm{PCV}^{25,26}$ Further investigations are needed to address the issue.

In conclusion, the presence of a fine vascular network within the polypoidal lesions in eyes with PCV may be an indicator of the activity of the lesion and their response to PDT. Our findings indicate that patients with a persistent, or enlarging fine vascular network after PDT need to be followed more frequently to detect early signs of a recurrence. The significance of the fine vascular network in the development and the course of PCV should be confirmed in a prospective study with a larger number of eyes.

\section{Summary}

\section{What was known before}

- Polypoidal choroidal vasculopathy responds well to photodynamic therapy, but the recurrences are not rare, and there was no way to estimate the possibility of recurrence.

\section{What this study adds}

- A component of fine vascular network was present as a part of the total network in most eyes with polypoidal choroidal vasculopathy. Although this network was reduced by photodynamic therapy, its subsequent enlargement was significantly associated with a recurrence of polypoidal vessels, and probably represents the active component of the lesion.

\section{Conflict of interest}

The authors declare no conflict of interest.

\section{Acknowledgements}

The study was supported in part by Grant-In-Aid for Scientific Research (No. 22791706) from the Japanese Society for the Promotion of Science, Tokyo, Japan.

\section{References}

1 Yannuzzi LA, Sorenson J, Spaide RF, Lipson B. Idiopathic polypoidal choroidal vasculopathy (IPCV). Retina 1990; 10(1): 1-8.
2 Rogers AH, Greenberg PB, Martidis A, Puliafito CA. Photodynamic therapy of polypoidal choroidal vasculopathy. Ophthalmic Surg Lasers Imaging 2003; 34(1): 60-63.

3 Spaide RF, Donsoff I, Lam DL, Yannuzzi LA, Jampol LM, Slakter J et al. Treatment of polypoidal choroidal vasculopathy with photodynamic therapy. Retina 2002; 22(5): 529-535.

4 Silva RM, Figueira J, Cachulo ML, Duarte L, Faria de Abreu JR, Cunha-Vaz JG. Polypoidal choroidal vasculopathy and photodynamic therapy with verteporfin. Graefes Arch Clin Exp Ophthalmol 2005; 243(10): 973-979.

5 Chan WM, Lam DS, Lai TY, Liu DT, Li KK, Yao Y et al. Photodynamic therapy with verteporfin for symptomatic polypoidal choroidal vasculopathy: one-year results of a prospective case series. Ophthalmology 2004; 111(8): 1576-1584.

6 Hussain N, Hussain A, Natarajan S. Role of photodynamic therapy in polypoidal choroidal vasculopathy. Indian J Ophthalmol 2005; 53(2): 101-104.

7 Lee SC, Seong YS, Kim SS, Koh HJ, Kwon OW. Photodynamic therapy with verteporfin for polypoidal choroidal vasculopathy of the macula. Ophthalmologica 2004; 218(3): 193-201.

8 Costa RA, Navajas EV, Farah ME, Calucci D, Cardillo JA, Scott IU. Polypoidal choroidal vasculopathy: angiographic characterization of the network vascular elements and a new treatment paradigm. Prog Retin Eye Res 2005; 24(5): 560-586.

9 Otani A, Sasahara M, Yodoi Y, Aikawa H, Tamura H, Tsujikawa A et al. Indocyanine green angiography: guided photodynamic therapy for polypoidal choroidal vasculopathy. Am J Ophthalmol 2007; 144(1): 7-14.

10 Wakabayashi T, Gomi F, Sawa M, Tsujikawa M, Tano Y. Marked vascular changes of polypoidal choroidal vasculopathy after photodynamic therapy. $\mathrm{Br} J$ Ophthalmol 2008; 92(7): 936-940.

11 Lee MW, Yeo I, Wong D, Ang CL. Photodynamic therapy with verteporfin for polypoidal choroidal vasculopathy. Eye 2008; 218(3): 193-201.

12 Lee WK, Lee PY, Lee SK. Photodynamic therapy for polypoidal choroidal vasculopathy: vaso-occlusive effect on the branching vascular network and origin of recurrence. Jpn J Ophthalmol 2008; 52(2): 108-115.

13 Akaza E, Mori R, Yuzawa M. Long-term results of photodynamic therapy of polypoidal choroidal vasculopathy. Retina 2008; 28(5): 717-722.

14 Kurashige Y, Otani A, Sasahara M, Yodoi Y, Tamura H, Tsujikawa A et al. Two-year results of photodynamic therapy for polypoidal choroidal vasculopathy. Am J Ophthalmol 2008; 146(4): 513-519.

15 Kang SW, Chung SE, Shin WJ, Lee JH. Polypoidal choroidal vasculopathy and late geographic hyperfluorescence on indocyanine green angiography. Br J Ophthalmol 2009; 93(6): 759-764.

16 Eandi CM, Ober MD, Freund KB, Slakter JS, Yannuzzi LA. Selective photodynamic therapy for neovascular age-related macular degeneration with polypoidal choroidal neovascularization. Retina 2007; 27(7): 825-831.

17 Sato T, Kishi S, Watanabe G, Matsumoto H, Mukai R. Tomographic features of branching vascular networks in polypoidal choroidal vasculopathy. Retina 2007; 27(5): 589-594.

18 Hikichi T, Ohtsuka H, Higuchi M, Matsushita T, Ariga H, Kosaka $\mathrm{S}$ et al. Improvement of angiographic findings of polypoidal choroidal vasculopathy after intravitreal injection of ranibizumab monthly for 3 months. Am J Ophthalmol 2010; 150(5): 674.e1-682.e1. 
19 Nakashizuka H, Mitsumata M, Okisaka S, Shimada H, Kawamura A, Mori R et al. Clinicopathologic findings in polypoidal choroidal vasculopathy. Invest Ophthalmol Vis Sci 2008; 49(11): 4729-4737.

20 Lafaut BA, Aisenbrey S, Van den Broecke C, Bartz-Schmidt KU, Heimann K. Polypoidal choroidal vasculopathy pattern in age-related macular degeneration: a clinicopathologic correlation. Retina 2000; 20(6): 650-654.

21 Rosa Jr RH, Davis JL, Eifrig CW. Clinicopathologic reports, case reports, and small case series: clinicopathologic correlation of idiopathic polypoidal choroidal vasculopathy. Arch Ophthalmol 2002; 120(4): 502-508.

22 Terasaki H, Miyake Y, Suzuki T, Nakamura M, Nagasaka T. Polypoidal choroidal vasculopathy treated with macular translocation: clinical pathological correlation. Br J Ophthalmol 2002; 86(3): 321-327.
23 Okubo A, Sameshima M, Uemura A, Kanda S, Ohba N. Clinicopathological correlation of polypoidal choroidal vasculopathy revealed by ultrastructural study. $\mathrm{Br} J$ Ophthalmol 2002; 86(10): 1093-1098.

24 Kuroiwa S, Tateiwa H, Hisatomi T, Ishibashi T, Yoshimura N. Pathological features of surgically excised polypoidal choroidal vasculopathy membranes. Clin Experiment Ophthalmol 2004; 32(3): 297-302.

25 Gomi F, Sawa M, Sakaguchi H, Tsujikawa M, Oshima Y, Kamei $\mathrm{M}$ et al. Efficacy of intravitreal bevacizumab for polypoidal choroidal vasculopathy. Br J Ophthalmol 2008; 92(1): 70-73.

26 Lai TY, Chan WM, Liu DT, Luk FO, Lam DS. Intravitreal bevacizumab (Avastin) with or without photodynamic therapy for the treatment of polypoidal choroidal vasculopathy. Br J Ophthalmol 2008; 92(5): 661-666. 